\title{
SEGETAL FLORA OF THE WIGRY NATIONAL PARK
}

\author{
${ }^{1}$ Teresa Skrajna, ${ }^{2}$ Helena Kubicka \\ ${ }^{1}$ The Agricultural Ecology Department of the University of Natural Sciences and Humanities \\ B. Prusa 14, 08-110 Siedlce, Poland \\ e-mail: tskrajna@op.pl \\ ${ }^{2}$ Polish Academy of Science, Botanical Garden - Center for Biological Diversity Conservation in Powsin, \\ Prawdziwka 2, 02-973 Warsaw, Poland
}

Received: 23.02.2011

\begin{abstract}
Segetal flora of the Wigry National Park (Poland) was studied in the period 2008-2010 within an area occupied by 33 villages. The analysis was based on 195 phytosociological relevés taken in fields of cereal crops, root plants and in stubble fields. The total number of species recorded in agrocenoses was 181 and they represented 36 botanical families. The most numerous families were Asteraceae, Fabaceae and Brasicaceae. The segetal flora of the Wigry National Park included mostly native species of apophytes $(53.6 \%)$ that predominated over anthropophytes. Among apophytes, meadow apophytes (35 species) and those from waterside habitats (26 species) were noted most often, archaeophytes (74 species) were predominant among anthropophytes, while epecophytes ( 9 species) and ergasiophytes (1 species) were much less abundant. Among the life forms analysed, therophytes $(61.9 \%)$ predominated over hemicryptophytes $(27.1 \%)$ and geophytes $(11 \%)$. The number of short-lived species in the flora of this Park was much greater $(68.5 \%)$ than that of perennial ones $(31.5 \%)$. The flora of the Park included 31 rare species classified in different categories of threat. Among them, Centaurium pulchellum and Centaurium erythraea belong to fully protected species (Dz.U. (Polish Journal of Laws) 2004 No. 168, item 1764).
\end{abstract}

Key words: segetal flora, anthropophytes, apophytes, rare species, Wigry National Park.

\section{INTRODUCTION}

The process of recession of many species has been observed in Poland for many years ( $\mathrm{Z}$ a r z y c $\mathrm{ki}$ and Kaźmierczakowa, 1993). It is also known to relate to a numerous group of segetal weeds (W a rcholińska, 1994a). Within the framework of a general strategy for nature conservation (L i r o, 2002; Programme Document of the Environmental
Protection Ministry, 2003), the most valuable components of agrocenoses are also protected. It seems that the protection of segetal weeds can be highly successful in national or landscape parks. Only a few reports have been devoted to the flora of agriculturally used fields within the areas of national parks or their protected zones (Trąba and Ziemińska, 1998; Jędruszc zak and Owczarczuk, 2006; S ołtys, 2006). In legally protected areas, crop fields are generally small and cultivated using traditional methods. They usually interact with plant communities characteristic of marshy, meadow, sward, forest and shrub habitats, which significantly enriches the flora of agrocenoses and allows conservation of a large group of weeds classified as rare at the regional or national level (Korniak, 1998; W a r c h o li ń s k a, 1994a). Agrophytocenoses are important components of the landscape of many national parks and occupy from 5\% to about $35 \%$ of their area (B o manow ska, 2006; R atuszniak and S o b i s z, 2006; S o ł t y s, 2006).

As yet, no detailed study on segetal flora in the area of the Wigry National Park (WNP) has been performed, although much attention has been devoted to the plants from natural habitats (Sokołowski, 1996). Segetal vegetation has been studied on a wide scale over the whole region of north-eastern Poland (K o r n i a k, 1992, 1998).

The main aim of the study was to determine the present state of segetal vegetation in the area of the Wigry National Park and its buffer zone. In particular, the study was undertaken to identify segetal flora species segetal flora most valuable for the region and the country, to find the sites of populations of rare and threatened species and to estimate their abundance. 


\section{THE STUDY AREA}

The Wigry National Park was founded in 1989 in order to protect a valuable complex of lakes and forest ecosystems in the area of the former landscape park. WPN is located in the south-eastern part of the Suwałki administrative unit. The park includes the northern part of the Augustów Primeval Forest, which is the largest compact forest complex in European Lowlands. According to the physiographic division (Kondracki, 2002), the Park's area comprises parts of three mesoregions: Western Suwałki Lake District, Eastern Suwałki Lake District and Augustów Plain. Together with the buffer zone, the Park spreads between $53^{\circ} 57^{\prime}$ and $54^{\circ} 10^{\prime}$ north latitude and $22^{\circ} 57^{\prime}$ and $23^{\circ} 15^{\prime}$ east longitude.

The area of the Park is $15,085.5$ ha, of which the greatest part is covered with forests $-9,464.5$ ha $(65.7 \%)$, while water occupies $2,907.2$ ha $(19.3 \%)$ and agriculturally used land occupies 2228 ha (14.8\%). The area of the buffer zone is $11,283.8$ ha (D a n o w $\mathrm{ski}$ et al. 2003). As far as morphology is concerned, the Park's terrain is much diversified and it formed as a result of Vistulian glaciation. To the north of Lake Wigry, there are hills of terminal moraines and pressed moraines cut across with many river valleys and lakes, while in the southern part gently rolling sandur plains are predominant.

The Park area lies in the middle part of the Czarna Hańcza River and belongs to the catchment area of the Niemen River, in the range of continental climate. It is the coolest part of Poland; the growing period lasts 175 days and the period with no ground frost is 30 days shorter than in central Poland. Mean annual precipitation is $650 \mathrm{~mm}$.

The soils in the Park area, formed from young glacier sedimentary rocks, are not very diverse. The largest area is covered with brown soils and pararendzinas characterised by a high content of calcium carbonate and skeleton particles, while podsolic soil as well as lessives and silt-peat soils are rare. A considerable part of the area covered by such soils is overgrown with natural vegetation and the soil in arable fields belongs to the poorest agricultural soil complexes. The soils ensuring higher production of the very good rye complex, good wheat complex and cereal-fodder strong complex are found mostly in the central part of the Park, for instance near the villages of Leszczewo, Leszczewek, Magdalenowo, Mikołajewo, Czerwony Folwark, Rosochaty Róg, and Maćkowa Ruda.

\section{MATERIALS AND METHODS}

The study on the segetal flora of the Wigry National Park and its agriculturally used buffer zone, whose results are presented in this paper, was conducted in the period 2008-2010. Within the area occupied by 33 villages (see Fig. 1), a series of 195 phytosociological relevés were taken in agrocenoses of cereals and root plants according to the well-known Braun-Blanquet method (P a w łow sk i, 1972) as well as a large number of floristic lists were made.

The taxonomic system of species followed Rutkowski (2007), while the nomenclature was adopted from Mir e k et al. (2002). Using the studies of Kornaś (1968), Korniak (1992), Jackowiak (1990), Zając E.U. and Zając A. (1975, 1992), Zając A. (1979) Zając et al. (1998) and Rutkowski (2007), a detailed analysis of the specimens was made taking into account the following criteria (see Table 1):

- persistence ( $\mathrm{S}$ - short lived, $\mathrm{P}$ - perennials)

- life form according to Raunkiaer ( $\mathrm{Ch}$ - chamaephytes, H- hemicryptophytes, G - geophytes, $\mathrm{T}$ - therophytes)

- geographic-historical group (A - apophytes: $\mathrm{m}$ - meadow, $\mathrm{w}$ - waterside, $\mathrm{ps}$ - psammophilous sward, $\mathrm{x}-$ xerothermic sward, $\mathrm{f}-$ forest, s - shrub; Ar - archaeophytes, Ep - epecophytes, Er - ergasiophygophytes)

- frequency of occurrence species according to conventional frequency scale $(1,2$ localities very rare; 3-7 localities - rare, 8-17 - frequent, 18-23-common)

- category of threat (Korniak, 1998; W ar cholińska, 1994; Zarzycki and Sze 1 ą g , 2006) (E-endangered with extinction, $\mathrm{V}$ - vulnerable, $\mathrm{R}$ - rare, I - indeterminate category of threat).

- conservation status according to the Decree of the Environmental Protection Minister of July $28^{\text {th }}, 2004$.

The distribution of sites the most interesting species in the investigated area is given in Fig. 5. 


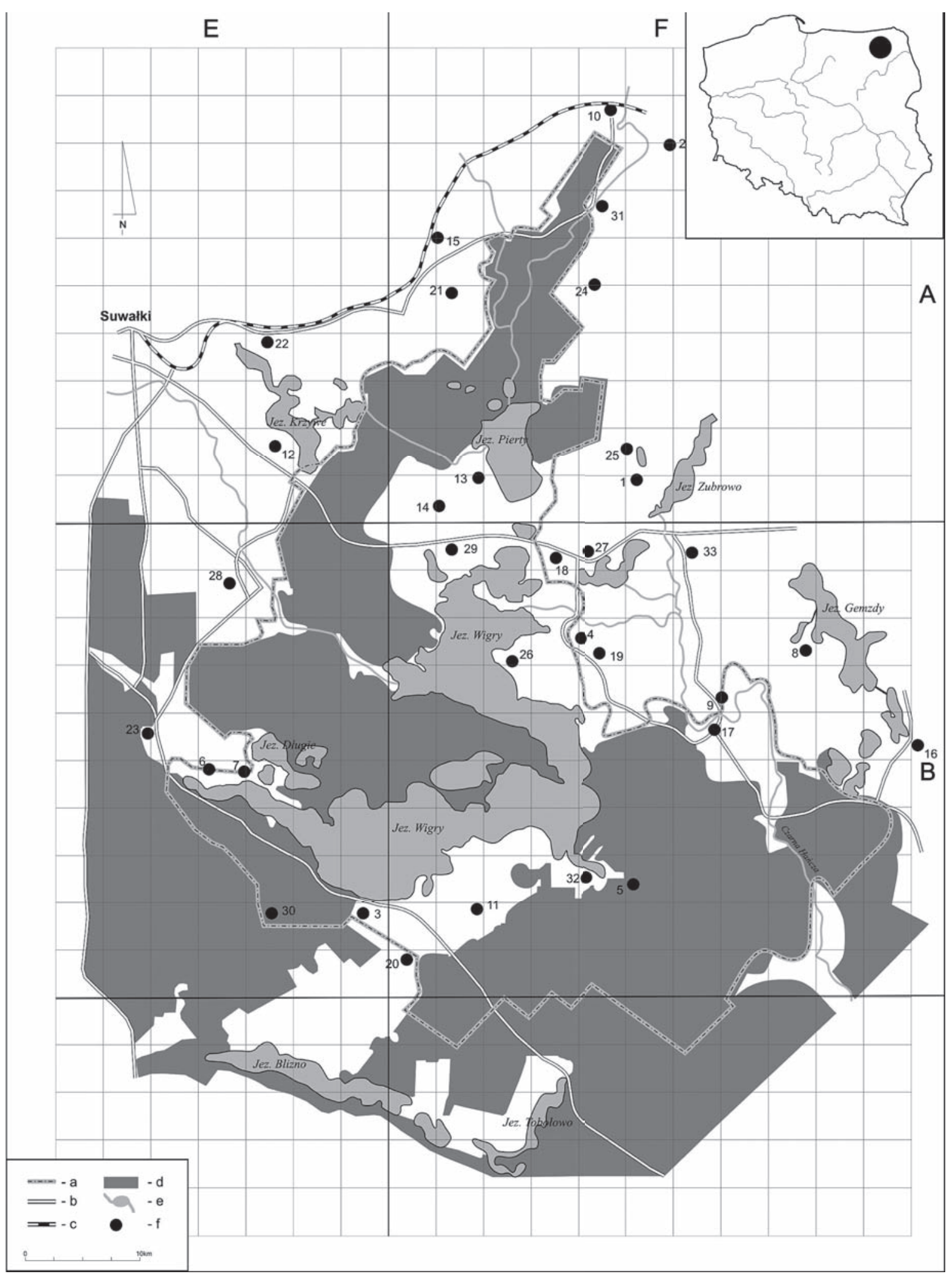

Fig. 1. The study area

$\mathrm{a}$ - borders of the Wigry National Park; b - road; c - railway; $\mathrm{d}$ - forest complexes; e - water, $\mathrm{f}$ - localities, study sites. 1 Aleksandrowo, 2. Bobrowisko, 3. Bryzgel, 4. Czerwony Folwark, 5. Czerwony Krzyż, 6. Gawrych Ruda, 7. Gaworzec, 8. Gremzdy Polskie, 9. Gremzdówka, 10. Kaletnik, 11. Krusznik, 12. Krzywe, 13. Leszczewo, 14. Leszczewek, 15. Lipniak, 16. Jeziorki, 17. Maćkowa Ruda, 18. Magdalenowo, 19. Mikołajewo, 20. Mońkinie, 21. Nowa Wieś, 22. Okuniowiec, 23. Płociczno, 24. Piotrowa Dąbrowa, 25. Ramienkiń26. Rosochaty Róg, 27. Ryżówka, 28. Sobolewo, 29. Stary Folwark, 30. Tartak, 31. Wiatrołuża 32. Zakąty, 33. Żubrówka Nowa 
Table 1.

Systematic list of species and characteristics of segetal flora of the Wigierski National Park

\begin{tabular}{|c|c|c|c|c|c|c|c|}
\hline Species & $\begin{array}{l}\text { Geographic- } \\
\text {-historical } \\
\text { groups }\end{array}$ & $\begin{array}{l}\text { Persi- } \\
\text { stence }\end{array}$ & $\begin{array}{l}\text { Life- } \\
\text {-form }\end{array}$ & $\begin{array}{l}\text { Frequency } \\
\text { occurrence }\end{array}$ & $\begin{array}{l}\text { Rare and critically } \\
\text { endangered species } \\
\text { in Poland }\end{array}$ & $\begin{array}{l}\text { Pro- } \\
\text { tected } \\
\text { species }\end{array}$ & $\begin{array}{l}\text { Regio- } \\
\text { nally rare } \\
\text { in N-E } \\
\text { Poland }\end{array}$ \\
\hline & & & & & 2 & 3 & 4 \\
\hline \multicolumn{8}{|l|}{ Equisetaceae } \\
\hline 1. Equisetum arvense L. & $\mathrm{Am}$ & $\mathrm{P}$ & G & common & & & \\
\hline 2. E. sylvaticum $\mathrm{L}$. & $\mathrm{Am}$ & $\mathrm{P}$ & G & frequent & & & \\
\hline \multicolumn{8}{|l|}{ Urticaceae } \\
\hline 3. Urtica urens L. & $\mathrm{Ar}$ & $\mathrm{S}$ & $\mathrm{T}$ & very rare & & & \\
\hline \multicolumn{8}{|l|}{ Polygonaceae } \\
\hline 4. Rumex crispus L. & $\operatorname{Am}(w)$ & $\mathrm{P}$ & G & very rare & & & \\
\hline 5. R. acetosella $\mathrm{L}$. & Aps & $\mathrm{P}$ & G & frequent & & & \\
\hline 6. R. acetosa $\mathrm{L}$. & Am & $\mathrm{P}$ & $\mathrm{H}$ & rare & & & \\
\hline 7. Polygonum amphibium L. & Aw & $\mathrm{P}$ & G & very rare & & & \\
\hline 8. P. persicaria $\mathrm{L}$. & Aw & $\mathrm{S}$ & $\mathrm{T}$ & frequent & & & \\
\hline $\begin{array}{l}\text { 9. P. lapathifolium L. subsp. pallidum / } \\
\text { With./ Fr. }\end{array}$ & Aw & $\mathrm{S}$ & $\mathrm{T}$ & common & & & \\
\hline 10. P. lapathifolium L. subsp. lapathifolium & Aw & $\mathrm{S}$ & $\mathrm{T}$ & common & & & \\
\hline 11. P. hydropiper $\mathrm{L}$. & Aw & S & $\mathrm{T}$ & rare & & & \\
\hline 12. P. aviculare $\mathrm{L}$. & Aw & S & $\mathrm{T}$ & frequent & & & \\
\hline 13. P. minus Huds. & Aw & $\mathrm{S}$ & $\mathrm{T}$ & rare & & & \\
\hline 14. Fallopia convolvulus /L./A. Löve & Ar & $\mathrm{S}$ & $\mathrm{T}$ & common & & & \\
\hline 15. Fagopyrum tataricum L. & Er & $\mathrm{S}$ & $\mathrm{T}$ & very rare & & & \\
\hline Chenopodiaceae & & $\mathrm{S}$ & & & & & \\
\hline 16. Chenopodium polyspermum $\mathrm{L}$. & Aw & $\mathrm{S}$ & $\mathrm{T}$ & rare & I & & I \\
\hline 17. Ch. album L. & Aw & $\mathrm{S}$ & $\mathrm{T}$ & common & & & \\
\hline 18. Atriplex patula L. & $\mathrm{Ar}$ & $\mathrm{S}$ & $\mathrm{T}$ & rare & & & \\
\hline Amaranthaceae & & $\mathrm{S}$ & & & & & \\
\hline 19. Amaranthus retroflexus L. & Ep & $\mathrm{S}$ & $\mathrm{K}$ & rare & & & \\
\hline 20. A. lividus L. & Ep & $\mathrm{S}$ & $\mathrm{T}$ & very rare & & & \\
\hline Caryophyllaceae & & $\mathrm{S}$ & & & & & \\
\hline 21. Arenaria serpyllifolia $\mathrm{L}$. & Ax & S & $\mathrm{T}$ & common & & & \\
\hline 22. Stellaria media /L./ Vill. & Am (f) & $\mathrm{S}$ & $\mathrm{T}$ & common & & & \\
\hline 23. S. graminea $\mathrm{L}$. & Am & $\mathrm{P}$ & $\mathrm{H}$ & rare & & & \\
\hline 24. Cerastium arvense L. & Aps & $\mathrm{P}$ & $\mathrm{H}(\mathrm{Ch})$ & rare & & & \\
\hline 25. C. holosteoides Fr. Em. Hyl. & Am & S & $\mathrm{H}$ & rare & & & \\
\hline 26. Sagina procumbens L. & $\operatorname{Am}(w)$ & $\mathrm{P}$ & $\mathrm{H}$ & rare & & & \\
\hline 27. Scleranthus annuus L. & $\mathrm{Ar}$ & $\mathrm{S}$ & $\mathrm{T}$ & frequent & & & \\
\hline 28. Herniaria glabra L. & Aps & S & $\mathrm{T}$ & rare & I & & I \\
\hline 29. Spergula arvensis L. & Ar & S & $\mathrm{T}$ & frequent & & & \\
\hline 30. Spergularia rubra/L./J. Presl et C. Presl & Aw & S & $\mathrm{T}$ & rare & & & I \\
\hline 31. Agrostemma githago L. & Ar & $\mathrm{S}$ & $\mathrm{T}$ & frequent & $\mathrm{V}$ & & $\mathrm{V}$ \\
\hline 32. Melandrium album /Mill./ Garcke & Am & $\mathrm{S}$ & $\mathrm{T}$ & frequent & & & \\
\hline 33. M. noctiflorum (L.) Fries & Ar & $\mathrm{S}$ & $\mathrm{T}$ & frequent & I & & \\
\hline
\end{tabular}




\begin{tabular}{|c|c|c|c|c|c|c|c|}
\hline 34. Silene vulgaris (Moench) Garcke & Aw & $\mathrm{S}$ & $\mathrm{T}$ & rare & & & \\
\hline 35. Gypsophila muralis L. & Aw & $\mathrm{S}$ & $\mathrm{T}$ & rare & & & $\mathrm{R}$ \\
\hline Ranunculaceae & & $\mathrm{S}$ & & & & & \\
\hline 36. Myosurus minimus L. & Aw & $\mathrm{S}$ & $\mathrm{T}$ & rare & I & $\mathrm{V}$ & I \\
\hline 37. Consolida regalis Gray & $\mathrm{Ar}$ & $\mathrm{S}$ & $\mathrm{T}$ & frequent & $\mathrm{I}$ & & \\
\hline 38. Ranunculus repens L. & $\mathrm{Am}(\mathrm{w})$ & $\mathrm{P}$ & $\mathrm{H}$ & frequent & & & \\
\hline
\end{tabular}

\section{Papaveraceae}

39. Papaver argemone $\mathrm{L}$.

40. P. dubium L.

41. P. rhoeas $\mathrm{L}$.

\section{Fumariaceae}

42. Fumaria officinalis L.

\section{Brassicaceae}

43. Sisymbrium officinale /L./ Scop.

44. Descurainia sophia/L./ Webb ex Prantl

45. Erysimum cheiranthoides L.

46. Arabidopsis thaliana/L./ Heynh.

47. Sinapis arvensis L.

48. Erophila verna /L./ Chevall.

49. Camelina microcarpa Andrz

50. Neslia paniculata (L.) Desv.

51. Capsella bursa-pastoris /L./ Medik.

52. Thlaspi arvense $\mathrm{L}$.

53. Armoracia rusticana P.Gaertn.,B.Mey. et Scherb.

54. Cardaminopsis arenosa/L./ Hayek

55. Raphanus raphanistrum $\mathrm{L}$.

56. Berteroa incana (L.) DC.

57. Rorippa sylvestris (L.) Besser

58. R. palustris (L.) Besser

\section{Rosaceae}

59. Potentilla anserina $\mathrm{L}$.

60. P. reptans $\mathrm{L}$.

61. P. norvegica L.

$\begin{array}{lllll}\text { Ar } & \mathrm{S} & \mathrm{T} & \text { rare } & \mathrm{V} \\ \mathrm{Ar} & \mathrm{S} & \mathrm{T} & \text { rare } & \mathrm{I} \\ \mathrm{Ar} & \mathrm{S} & \mathrm{T} & \text { rare } & \end{array}$

62. Geum urbanum L.

\section{Fabaceae}

63. Vicia sativa $\mathrm{L}$.

64. V. angustifolia Scop.

65. V. hirsuta /.L/ S.F. Gray

66. V. tetrasperma L.

67. V. villosa $\mathrm{L}$.

68. V. cracca L.

69. Lathyrus pratensis L.

70. L. tuberosus L.

71. Melilotus alba Medik.

72. Medicago lupulina L.

$\begin{array}{ccccc}\text { Ar } & \mathrm{S} & \mathrm{T} & \text { rare } & \\ \mathrm{Ar} & \mathrm{S} & \mathrm{T} & \text { frequent } & \\ \mathrm{Ar} & \mathrm{S} & \mathrm{T} & \text { common } & \\ \mathrm{Ar} & \mathrm{S} & \mathrm{T} & \text { frequent } & \\ \mathrm{Ar} & \mathrm{S} & \mathrm{T} & \text { frequent } & \\ \mathrm{Am} & \mathrm{P} & \mathrm{H} & \text { rare } & \\ \mathrm{Am} & \mathrm{P} & \mathrm{H} & \text { rare } & \\ \mathrm{Ar} & \mathrm{P} & \mathrm{G} & \text { very rare } & \mathrm{R} \\ \mathrm{Ar} & \mathrm{S} & \mathrm{H} & \text { frequent } & \\ \mathrm{Ax} & \mathrm{S} & \mathrm{T}(\mathrm{H}) & \text { rare } & \end{array}$


73. Trifolium arvense $\mathrm{L}$

74. T. dubium Sibth.

75 T. repens $\mathrm{L}$.

76 T. pratense $\mathrm{L}$.

77. T. hybridum L.

78. T. medium L.

79. T. montanum L.

80. Anthyllis vulneraria L.

81. Lotus corniculatus L.

\section{Oxalidaceae}

82. Oxalis fontana Bunge

\section{Geraniaceae}

83. Geranium dissectum L.

84. G. pusillum Burm. F. ex L.

85. Erodium cicutarium /L./ L

\section{Euphorbiaceae}

86. Euphorbia peplus L.

87. E. helioscopia L.

88. E. esula L.

\begin{tabular}{|c|c|c|c|c|c|c|c|}
\hline 88. E. esula L. & Ax & $\mathrm{S}$ & $\mathrm{T}$ & very rare & & & \\
\hline Malvaceae & & $\mathrm{S}$ & & & & & \\
\hline 89. Malva neglecta Wallr. & $\mathrm{Ar}$ & $\mathrm{S}$ & $\mathrm{T}$ & very rare & & & \\
\hline 90. M. alcea $\mathrm{L}$. & $\mathrm{Ar}$ & $\mathrm{S}$ & $\mathrm{H}$ & very rare & & & \\
\hline \multicolumn{8}{|l|}{ Clusiaceae } \\
\hline 91. Hypericum perforatum $\mathrm{L}$. & Am & $\mathrm{P}$ & $\mathrm{H}$ & rare & & & \\
\hline \multicolumn{8}{|l|}{ Violaceae } \\
\hline 92. Viola arvensis Murray & $\mathrm{Ar}$ & $\mathrm{S}$ & $\mathrm{T}$ & common & & & \\
\hline 93. V. tricolor $\mathrm{L}$. & $\mathrm{Ar}$ & $\mathrm{S}$ & $\mathrm{T}$ & rare & & & $\mathrm{R}$ \\
\hline \multicolumn{8}{|l|}{ Onagraceae } \\
\hline 94. Epilobium parviflorum Schreb. & Aw & $\mathrm{P}$ & $\mathrm{H}$ & frequent & & & \\
\hline 95. E. roseum Schreber & Aw & $\mathrm{P}$ & $\mathrm{H}$ & rare & & & \\
\hline \multicolumn{8}{|l|}{ Apiaceae } \\
\hline 96. Pimpinella saxifraga $\mathrm{L}$. & Am & $\mathrm{P}$ & $\mathrm{H}$ & rare & & & \\
\hline 97. Aethusa cynapium $\mathrm{L}$. & $\mathrm{Ar}$ & $\mathrm{S}$ & $\mathrm{T}$ & frequent & I & & I \\
\hline 98. Torilis japonica (Houtt.) DC & As & $\mathrm{S}$ & $\mathrm{T}(\mathrm{H})$ & very rare & & & \\
\hline 99. Daucus carota L. & $\mathrm{Ar}$ & $\mathrm{S}$ & $\mathrm{T}$ & frequent & & & \\
\hline Primulaceae & & $\mathrm{S}$ & & & & & \\
\hline 100. Anagallis arvensis L. & $\mathrm{Ar}$ & $\mathrm{S}$ & $\mathrm{T}$ & frequent & & & \\
\hline 101. Lysimachia nummularia L. & Am & $\mathrm{P}$ & G & rare & & & \\
\hline 102. Centunculus minimus L. & $\mathrm{Ar}$ & $\mathrm{S}$ & $\mathrm{T}$ & very rare & $\mathrm{V}$ & & $\mathrm{V}$ \\
\hline Gentianaceae & & $\mathrm{S}$ & & & & & \\
\hline 103. Centaurium erythraea Rafn & $\mathrm{Am}$ & $\mathrm{S}$ & $\mathrm{H}$ & very rare & & $\mathrm{Rc}$ & \\
\hline 104. C. pulchellum (Sw.) Druce & Aw & $\mathrm{S}$ & $\mathrm{T}$ & very rare & $\mathrm{V}$ & $\mathrm{Rc}$ & $\mathrm{V}$ \\
\hline Rubiaceae & & $\mathrm{S}$ & & & & & \\
\hline 105. Galium aparine L. & Af & $\mathrm{S}$ & $\mathrm{T}$ & common & & & \\
\hline \multicolumn{8}{|l|}{ Convolvulaceae } \\
\hline 106. Convolvulus arvensis L. & $\mathrm{Ax}$ & $\mathrm{P}$ & $\mathrm{G}$ & common & & & \\
\hline 107. Cuscuta europaea L. & $\mathrm{Ar}$ & $\mathrm{S}$ & $\mathrm{T}$ & very rare & $\mathrm{V}$ & & $\mathrm{V}$ \\
\hline
\end{tabular}

$\begin{array}{llcc}\text { Aps } & \mathrm{S} & \mathrm{T} & \text { rare } \\ \mathrm{Am} & \mathrm{S} & \mathrm{T} & \text { rare } \\ \mathrm{Am} & \mathrm{P} & \mathrm{H} & \text { frequent } \\ \mathrm{Am} & \mathrm{S} & \mathrm{T} & \text { rare } \\ \mathrm{Am} & \mathrm{P} & \mathrm{H} & \text { rare } \\ \mathrm{Az} & \mathrm{P} & \mathrm{H} & \text { rare } \\ \mathrm{Az} & \mathrm{P} & \mathrm{H} & \text { rare } \\ \mathrm{Ax} & \mathrm{P} & \mathrm{H} & \text { very rare } \\ \mathrm{Am} & \mathrm{S} & \mathrm{T} & \text { rare }\end{array}$

Ep $\quad$ P G frequent

$\begin{array}{lllll}\mathrm{Ar} & \mathrm{S} & \mathrm{T} & \text { very rare } & \mathrm{V}\end{array}$

Ar $\quad S \quad T \quad$ common

Ar $\quad \mathrm{S} \quad \mathrm{T}(\mathrm{H})$ frequent

Ar $\quad S \quad T$ very rare

Ax $\quad \mathrm{G}(\mathrm{H})$ frequent

$\begin{array}{llll}\text { Ax } & P & \mathrm{G}(\mathrm{H}) & \text { frequent }\end{array}$ 


\begin{tabular}{|c|c|c|c|c|c|c|c|}
\hline Boraginaceae & & & & & & & \\
\hline 108. Lithospermum arvense $\mathrm{L}$. & $\mathrm{Ar}$ & $\mathrm{S}$ & $\mathrm{T}$ & rare & & & \\
\hline 109. Echium vulgare L. & $\mathrm{Ar}$ & S & $\mathrm{H}$ & frequent & & & \\
\hline 110. Anchusa arvensis (L.) M. Bieb. & $\mathrm{Ar}$ & $\mathrm{S}$ & $\mathrm{H}$ & frequent & & & \\
\hline 111. Anchusa officinalis L. & $\mathrm{Ar}$ & $\mathrm{S}$ & $\mathrm{H}$ & frequent & & & \\
\hline 112. Myosotis arvensis /L./ Hill. & $\mathrm{Ar}$ & $\mathrm{S}$ & $\mathrm{T}$ & frequent & & & \\
\hline 113. M. stricta Link ex Roem. et Schult. & Aps & $\mathrm{S}$ & $\mathrm{T}$ & very rare & & & \\
\hline 114. Asperugo procumbens L. & Ep & $\mathrm{S}$ & $\mathrm{T}$ & very rare & $\mathrm{V}$ & $\mathrm{E}$ & $\mathrm{V}$ \\
\hline Solanaceae & & $\mathrm{S}$ & & & & & \\
\hline 115. Solanum nigrum L. em. Mill. & $\mathrm{Ar}$ & $\mathrm{S}$ & $\mathrm{T}$ & frequent & & & \\
\hline \multicolumn{8}{|l|}{ Scrophulariaceae } \\
\hline 116. Linaria vulgaris Mill. & Aps & $\mathrm{P}$ & G & rare & & & \\
\hline 117. Chaenorhinum minus /L./ Lange & Ax & $\mathrm{S}$ & $\mathrm{T}$ & frequent & $\mathrm{R}$ & & $\mathrm{R}$ \\
\hline 118. Veronica serpyllifolia L. & Am & $\mathrm{P}$ & $\mathrm{H}$ & rare & & & \\
\hline 119. V. arvensis L. & $\mathrm{Ar}$ & $\mathrm{S}$ & $\mathrm{T}$ & common & & & \\
\hline 120. V. opaca Fr. & Ar & $\mathrm{S}$ & $\mathrm{T}$ & rare & $\mathrm{V}$ & & \\
\hline 121. V. persica Poir. & Ep & $\mathrm{S}$ & $\mathrm{T}$ & frequent & & & \\
\hline 122. V. agrestis $\mathrm{L}$. & Ar & $\mathrm{S}$ & $\mathrm{T}$ & rare & I & & \\
\hline 123. Rhinanthus serotinus (Schönh.) Oborný & $\mathrm{Ar}$ & $\mathrm{S}$ & $\mathrm{T}$ & frequent & & & \\
\hline \multicolumn{8}{|l|}{ Lamiaceae } \\
\hline 124. Scutellaria galericulata L. & Aw & $\mathrm{P}$ & $\mathrm{H}$ & very rare & & & \\
\hline 125. Galeopsis pubescens Besser. & Af & $\mathrm{S}$ & $\mathrm{T}$ & rare & & & \\
\hline 126. G. tetrahit L. & Af & S & $\mathrm{T}$ & common & & & \\
\hline 127. G. bifida Boenn. & Af & $\mathrm{S}$ & $\mathrm{T}$ & rare & & & \\
\hline 128. Stachys annua (L.) L. & $\mathrm{Ar}$ & S & $\mathrm{T}$ & frequent & $\mathrm{V}$ & & $\mathrm{V}$ \\
\hline 129. S. palustris L. & Af & $\mathrm{P}$ & G & frequent & & & \\
\hline 130. Glechoma hederacea L. & Am & $\mathrm{P}$ & $\mathrm{H}$ & rare & & & \\
\hline 131. Prunella vulgaris L. & Am & $\mathrm{P}$ & $\mathrm{H}$ & rare & & & \\
\hline 132. Lamium purpureum L. & $\mathrm{Ar}$ & S & $\mathrm{T}$ & common & & & \\
\hline 133. L. amplexicaule $\mathrm{L}$. & $\mathrm{Ar}$ & $\mathrm{S}$ & $\mathrm{T}$ & frequent & & & \\
\hline 134. Acinos arvensis (Lam.) Dandy & $\mathrm{Ax}$ & $\mathrm{S}$ & $\mathrm{H}$ & rare & I & & \\
\hline 135. Mentha arvensis L. & Aw & $\mathrm{P}$ & $\mathrm{H}$ & frequent & & & \\
\hline 136. Elsholtzia ciliata (Thunb.) Hyl. & Ep & $\mathrm{S}$ & $\mathrm{T}$ & very rare & & & \\
\hline \multicolumn{8}{|l|}{ Plantaginaceae } \\
\hline 137. Plantago major L. & Af & $\mathrm{P}$ & $\mathrm{H}$ & frequent & & & \\
\hline 138. P. intermedia Gilib. & Aw & $\mathrm{P}$ & $\mathrm{H}$ & frequent & & & \\
\hline 139. P. lanceolata L. & $\mathrm{Am}$ & $\mathrm{P}$ & $\mathrm{H}$ & frequent & & & \\
\hline \multicolumn{8}{|l|}{ Valerianaceae } \\
\hline 140. Valerianella dentata (L.) Poll. & $\mathrm{Ar}$ & $\mathrm{S}$ & $\mathrm{T}$ & very rare & $\mathrm{I}$ & & $\mathrm{R}$ \\
\hline \multicolumn{8}{|l|}{ Dipsacaceae } \\
\hline 141. Knautia arvensis (L.)J. M. Coult. & Af & $\mathrm{P}$ & $\mathrm{H}$ & frequent & & & \\
\hline \multicolumn{8}{|l|}{ Companulaceae } \\
\hline 142. Campanula rapunculoides L. & $\mathrm{Ax}$ & $\mathrm{P}$ & G & frequent & I & & \\
\hline \multicolumn{8}{|l|}{ Asteraceae } \\
\hline 143. Conyza canadensis /L./ Cronquist & $\mathrm{Ep}$ & $\mathrm{S}$ & $\mathrm{T}$ & common & & & \\
\hline 144. Gnaphalium uliginosum L. & Aw & $\mathrm{S}$ & $\mathrm{T}$ & frequent & & & \\
\hline
\end{tabular}




\begin{tabular}{|c|c|c|c|c|c|}
\hline 145. Bidens tripartita $\mathrm{L}$. & Aw & $\mathrm{S}$ & $\mathrm{T}$ & frequent & \\
\hline 146. Galinsoga parviflora Cav. & Ep & $\mathrm{S}$ & $\mathrm{T}$ & frequent & \\
\hline 147. G. ciliata /Raf./S.F. Blake & Ep & $\mathrm{S}$ & $\mathrm{T}$ & frequent & \\
\hline 148. Anthemis tinctoria $\mathrm{L}$. & $\mathrm{Ar}$ & $\mathrm{S}$ & $\mathrm{H}$ & frequent & $\mathrm{R}$ \\
\hline 149. Anthemis arvensis L. & $\mathrm{Ar}$ & $\mathrm{S}$ & $\mathrm{T}$ & common & \\
\hline 150. Achillea millefolium $\mathrm{L}$. & Am & $\mathrm{P}$ & $\mathrm{H}$ & frequent & \\
\hline 151. Chamomilla recutita /L./ Rauschert & $\mathrm{Ar}$ & $\mathrm{S}$ & $\mathrm{T}$ & rare & \\
\hline $\begin{array}{l}\text { 152. Matricaria maritima L. subsp. inodora } \\
\text { /L./ Dostal }\end{array}$ & $\mathrm{Ar}$ & $\mathrm{S}$ & $\mathrm{T}$ & frequent & \\
\hline 153. Artemisia vulgaris L. & $\operatorname{Am}(w)$ & $\mathrm{P}$ & $\mathrm{H}$ & rare & \\
\hline 154. Tussilago farfara $\mathrm{L}$. & Am & $\mathrm{P}$ & G & rare & \\
\hline 155. Senecio vulgaris L. & $\mathrm{Ar}$ & $\mathrm{S}$ & $\mathrm{T}(\mathrm{H})$ & rare & \\
\hline 156. Arctium lappa L. & Af & $\mathrm{S}$ & $\mathrm{H}$ & rare & \\
\hline 157. Cirsium arvense /L./ Scop. & Af & $\mathrm{P}$ & G & common & \\
\hline 158. Centaurea cyanus L. & $\mathrm{Ar}$ & $\mathrm{S}$ & $\mathrm{T}$ & frequent & \\
\hline 159. Cichorium intybus L. & $\mathrm{Ar}$ & $\mathrm{P}$ & $\mathrm{H}$ & rare & \\
\hline 160. Leontodon autumnalis L. & Am & $\mathrm{P}$ & $\mathrm{H}$ & frequent & \\
\hline 161. Sonchus oleraceus L. & $\mathrm{Ar}$ & $\mathrm{S}$ & $\mathrm{T}(\mathrm{H})$ & frequent & \\
\hline 162 S. asper/L./ Hill & $\mathrm{Ar}$ & $\mathrm{S}$ & $\mathrm{T}$ & rare & \\
\hline 163. S. arvensis L. & $\mathrm{Ar}$ & $\mathrm{P}$ & $\mathrm{H}$ & frequent & \\
\hline 164. Lactuca serriola $\mathrm{L}$. & $\mathrm{Ar}$ & $\mathrm{S}$ & $\mathrm{H}$ & rare & \\
\hline 165. Taraxacum officinale F.H. Wigg. & Am & $\mathrm{P}$ & $\mathrm{H}$ & frequent & \\
\hline 166. Lapsana communis L. & Af & $\mathrm{S}$ & $\mathrm{T}(\mathrm{H})$ & frequent & \\
\hline
\end{tabular}

\begin{tabular}{|c|c|c|c|c|c|c|}
\hline \multicolumn{7}{|l|}{ Juncaceae } \\
\hline 167. Juncus bufonius L. & Aw & $\mathrm{S}$ & $\mathrm{T}$ & frequent & & \\
\hline \multicolumn{7}{|l|}{ Cyperaceae } \\
\hline 168. Carex hirta L. & Am & $\mathrm{P}$ & G & rare & & \\
\hline \multicolumn{7}{|l|}{ Poaceae } \\
\hline 169. Lolium perenne $\mathrm{L}$. & Am & $\mathrm{P}$ & $\mathrm{H}$ & frequent & & \\
\hline 170. Роа аппиа L. & Am & $\mathrm{S}$ & $\mathrm{T}$ & common & & \\
\hline 171. Apera spica-venti (L.) P. Beauv. & $\mathrm{Ar}$ & $\mathrm{S}$ & $\mathrm{T}$ & frequent & & \\
\hline 172. Bromus secalinus L. & $\mathrm{Ar}$ & $\mathrm{S}$ & $\mathrm{T}$ & frequent & V & $\mathrm{V}$ \\
\hline 173. Elymus repens (L.) Gould. & Aw & $\mathrm{P}$ & G & common & & \\
\hline 174. Avena strigosa Schreber & $\mathrm{Ar}$ & $\mathrm{S}$ & $\mathrm{T}$ & frequent & $\mathrm{E}$ & \\
\hline 175. A. fatua $\mathrm{L}$. & $\mathrm{Ar}$ & $\mathrm{S}$ & $\mathrm{T}$ & frequent & & \\
\hline 176. Agrostis gigantea Roth. & Am & $\mathrm{P}$ & $\mathrm{H}$ & frequent & & \\
\hline $\begin{array}{l}\text { 177. Phragmites australis (Cav.) Trin. } \\
\text { ex Steud. }\end{array}$ & Aw & $\mathrm{P}$ & G & rare & & \\
\hline 178. Echinochloa crus-galli /L./P. Beauv. & $\mathrm{Ar}$ & $\mathrm{S}$ & $\mathrm{T}$ & frequent & & \\
\hline $\begin{array}{l}\text { 179. Digitaria ischaemum /Schreb./ H.L. } \\
\text { Muhl. }\end{array}$ & $\mathrm{Ar}$ & $\mathrm{S}$ & $\mathrm{T}$ & frequent & & \\
\hline 180. Setaria viridis (L.)P.B. & $\mathrm{Ar}$ & $\mathrm{S}$ & $\mathrm{T}$ & frequent & & \\
\hline 181. S. pumila /Poir./ Poem et Schult. & $\mathrm{Ar}$ & $\mathrm{S}$ & $\mathrm{T}$ & frequent & & \\
\hline
\end{tabular}

Explanations: Ar - archaeophytes, Ep - epecophytes, Kn - kenophytes, A - Apophytes: m - meadow, w - waterside, ps - psammophilous sward, $\mathrm{x}$ - xerothermic sward, f - forest, s - shrub; S - short-lived, P - perennials, T - therophytes, $\mathrm{H}$ - hemicryptophytes, $\mathrm{G}$ - geophytes, $\mathrm{Ch}$ - chamaephytes,

1-Polish red list of segetal weeds, Warcholińska 1994, 2- Polish red list of vascular plants, Zarzycki and Szeląg 2006, 3 Conservation status 2004, 4- N-E Poland Korniak 1998. E - critically endangered R - rare, V - vulnerable, I - taxa of indeterminate category of threat. Rc- legal status - full protection. 


\section{RESULTS}

The segetal flora of the Wigry National Park was found to comprise 181 species of vascular plants representing 125 genera and 36 botanic families. The majority (62\%) of the flora represent 7 most abundant families of which the most richly represented were: Asteraceae (24 species and 20 genera), Fabaceae (19 species and 6 genera) and Brasicaceae (16 species and 15 genera). Over half of the species identified was found to be very rare or rare $(102-56 \%)$. These were mostly the species coming from the neighbouring forest and meadow or ruderal communities, such as: Pimpinella saxifraga, Rumex acetosa, Stellaria graminea, Lathyrus pratensis, Torilis japonica. As far the conservation status is concerned, endangered species (Rc) such as: Centaurium erythraea, Centaurium pulchellum and those on the national red lists of vulnerable species: Lathyrus tuberosus, Geranium dissectum, Centunculus minimus and others, were also rarely found. Typical weeds accompanying field crops, which are eurytopic taxa such as: Matricaria maritima subsp. inodora, Centaurea cyanus, Echinochloa crus-galli, Polygonum aviculare, were frequently encountered. Troublesome field weeds represented by 21 species and accounting for $11.6 \%$ of the segetal flora of the Park, such as: Chenopodium album, Polygonum lapathifolium subsp. lapathifolium, Fallopia convolvulus, Stellaria media, Viola arvensis, Cirsium arvense, Elymus repens and others were also frequent.

Analysis of the proportions of geographical-historical groups in the segetal flora of the Park revealed the predominance of native species and apophytes (96 species making up 53.6\% of the flora) over anthropophytes (Fig. 2). Species diversity of the apophytes reflects the habitat conditions of the area studied. The dominant species are apophytes of meadow habitats (35 species) and waterside habitats (26 species). The proportion of apophytes originating from forest and shrub habitats is also significant 16 species. The other apophytes found were characteristic of xerothermic grassland (11 species) and psammophylous grassland (9 species) (Fig. 3). Common apophytes are, e.g., Equisetum arvense, Polygonum lapathifolium subsp. lapathifolium, Arenaria serpyllifolia, whereas Herniaria glabra, Arabidopsis thaliana, Gypsophila muralis and others are met much less frequently.

Among anthrophytes, the most numerous group is that of archaeophytes (74 species), including troublesome weeds such as Viola arvensis, Anthemis arvensis, Cirsium arvense, as well as all protected, rare and threatened species in the country, such as: Agrostemma githago, Papaver argemone, Neslia paniculata, Centunculus minimus. The percentage of kenophytes is low (5.5\% of the flora); they are mainly represented by epecophytes ( 9 species) of which $O x a$ lis fontana, Veronica persica, Conyza canadensis and Galinsoga parviflora are the most frequently noted and which locally can be a threat to field crops. Ergasiophygophytes are represented only by Fagopyrum tataricum which is very rare in the Park area.

The segetal flora of the Wigry National Park is dominated by short-lived species (124 species, $68.5 \%$ of the flora) compared to perennial ones (57 species, $31.5 \%)$. From among the life forms analysed, terophytes (112 species, 61.9\%) are predominant over hemicryptophytes (49 species, 27.1\%) and geophytes (20 species, 11\%) (Fig. 4).

The flora of the Park's phytoagrocenoses includes 31 valuable species representing the conservation status of endangered, rare and legally protected species. On the basis of the red list of segetal species threatened in our country (W a r c h o l i ń s k a, 1994; Zarzycki and Szeląg, 2006), 30 species representing four categories of conservation status were identified:

E - (endangered) - Asperugo procumbens, Avena strigosa

$\mathrm{V}$ - (vulnerable) - Agrostemma githago, Bromus secalinus, Camelina microcarpa, Centunculus minimus, Centaurium pulchellum, Cuscuta europaea, Geranium dissectum, Papaver argemone, Stachys annua,Veronica opaca.

$\mathrm{R}$ - (rare) - Anthemis tinctoria, Chaenorhinum minus, Lathyrus tuberosus.

I - (indeterminate category of threat) - Aethusa cynapium ssp. agrestis, Acinos arvensis, Campanula rapunculoides, Chenopodium polyspermum, Consolida regalis, Fumaria officinalis, Herniaria glabra, Melandrium noctiflorum, Myosurus minimus, Neslia paniculata, Papaver dubium, P. rhoeas, Sinapis arvensis, Valerianella dentata, Veronica agrestis.

Moreover, the Park's flora was found to include some species from the regional red list (K or n i a k, 1998), such as: Spergularia rubra, Gypsophila muralis, and Viola tricolor. Centaurium pulchellum and Centaurium erythraea are fully protected species (Dz.U. (Polish Journal of Laws) 2004 No. 168, item 1764) that are encountered very rarely in the area studied. 


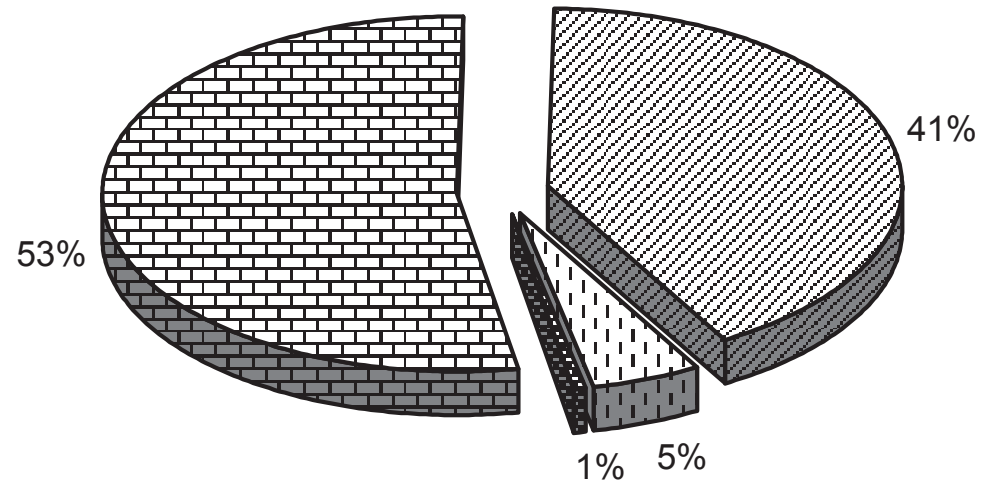

田 apophytes $\square$ archeophytes $\square$ epecophytes :

Fig. 2. Proportions of geographical-historical groups in the segetal flora in the area of the Wigry National Park.
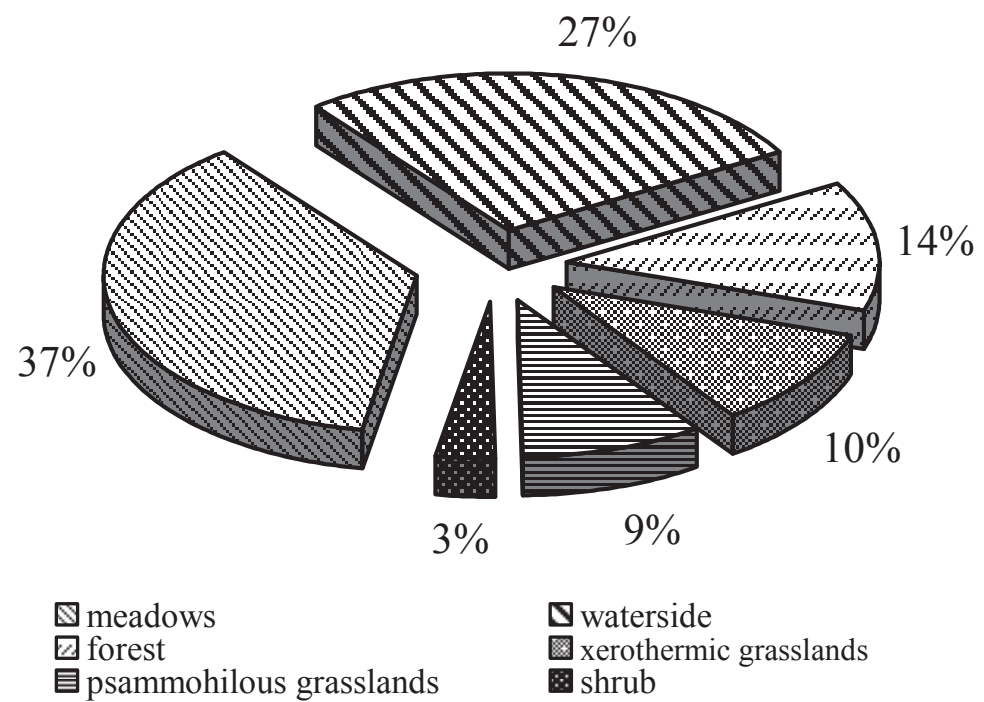

$\mathbf{\nabla}$ waterside

xerothermic grasslands shrub

Fig. 3. Origin of apophytes in the segetal flora in the area of the Wigry National Park.

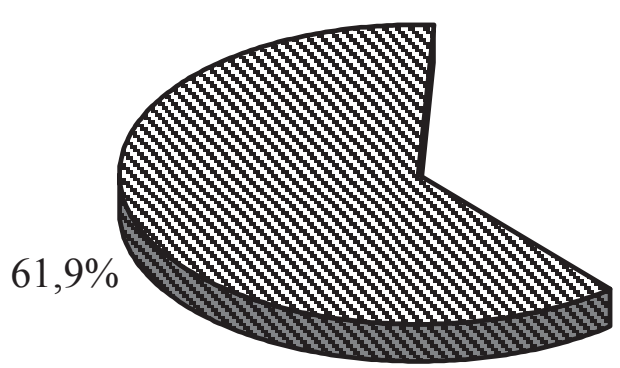

$\mathbb{Q}$ therophytes 㐁hemicryptophytes
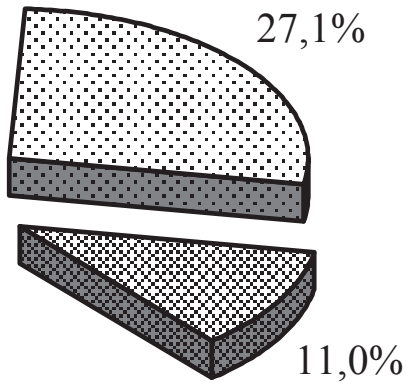

图 geophytes

Fig. 4. Proportions of different biological forms in the segetal flora in the area of the Wigry National Park. 


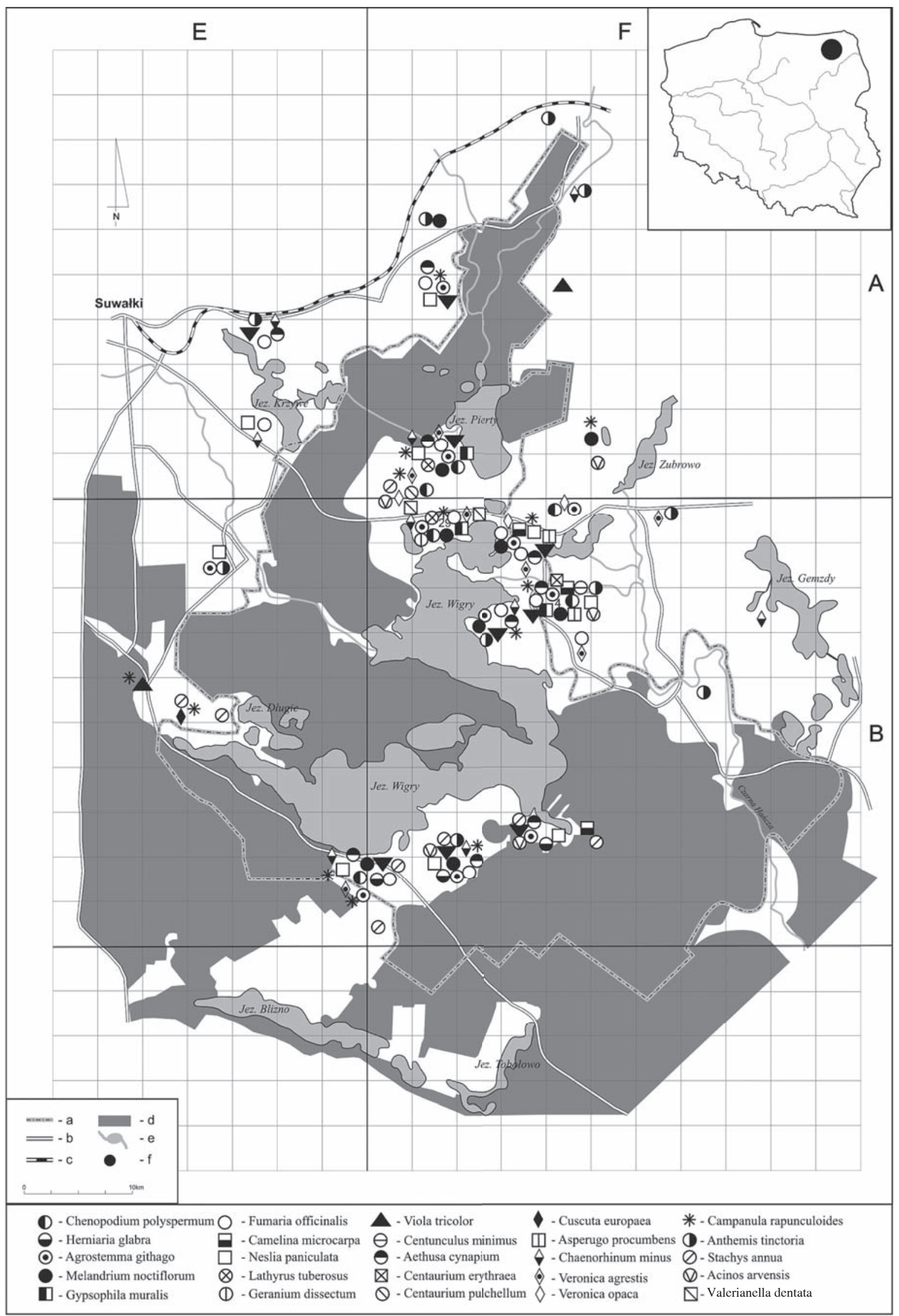

Fig. 5. Occurrence of rare species of the segetal flora within the borders of the Wigry National Park. 


\section{DISCUSSION}

The segetal flora of the Wigry National Park accounts for $51 \%$ of the flora of agrocenoses of north-eastern Poland (Korniak, 1992). Taking into account the small area occupied by agrocenoses in the Park and its buffer zone, it can be considered to be rather rich. The richness is mostly a consequence of the specific character of agriculture in the area (arable fields are usually small and tilled in a traditional way) and the diverse mosaic landscape of the Park related to diverse ecological potential of the field habitats. Such a specific form of agricultural land use is assumed by the National Strategy of Protection and Moderate Use of Biological Diversity as one of the measures aimed at conservation of the diversity of agrophytocenoses.

The main threat to segetal weeds in the protected area is cessation of agricultural use of the fields in this area. Changes in land use lead to changes in the species composition of the segetal flora and to restriction or disappearance of entire communities (B o m a n ow s ka, 2006; S o ł ty s, 2006).

The analysis of the segetal flora of the Wigry National Park shows that the contribution of geographically-historical groups, life forms and the persistence of species are similar to those in the majority of local segetal floras of protected areas (B o m a n o w ska, 2006; Gołdyn etal. 2002; Hołdyński and Korniak, 1994; Ratuszniak and Sobisz, 2006; S ołtys, 2006; Zi e mińska-S myk, 2006). An interesting feature is the presence of a large group of species of different conservation status. The species from this group represent $44.3 \%$ of total species with conservation status in the region (K o r $\mathrm{niak}$, 1998). This group of species includes those from the national red list (W a r ch olińs k a 1994a; Zarzycki and S ze lą g, 2006) and fully protected species (the Decree of the Environmental Protection Minister of July $28^{\text {th }}$, 2004).

The problem of impoverishment of segetal communities has been discussed in many papers and it concerns the flora of both protected areas (K or czyński, 2006; Rzymowska and Skrajna, 2006; S ok ołowski, 1989; S ołtys, 2006) and typical agriculturally used land (F i j a k k w s ki and Ny c z, 1998; K ornia k, 1992; S i c iń s ki , 2003; Wa rcholińska, 1994b). In view of the above and given the results of our study, it seems highly recommendable to monitor the segetal flora, in particular the rare species, in the area of the Wigry National Park in order to grasp the dynamics of changes in its structure and to conserve the species diversity of agrocenoses.

\section{REFERENCES}

B omanowska A., 2006. Wybrane cechy flory segetalnej Kampinowskiego Parku Narodowego/ Some features of the segetal flora of the Kampinos National Park. Pam. Puław., IUNG Puławy, 143: 27-35. (in Polish)

Danowski J., Danowska Małgorzata, M., Witýn ska S., 2003. Program ochrony środowiska powiatu suwalskiego na lata 2004-2012. / The environmental protection programme of Suwałki County for the period 2004-2012: 3-23. (in Polish)

Gołdyn H., Arczyńska-Chudy E., Kujawa A., Jezierska-Madziar M., 2002. Flora segetalna Parku krajobrazowego im. Gen. D. Chłapowskiego. / Segetal flora of the General D. Chłapowski Landscape Park. Acta Agrobot. 55 (2): 167-181. (in Polish)

Fijałkowski D., Nycz B., 1998. Zagrożone gatunki roślin segetalnych na Lubelszczyźnie. / Threatened species of segetal plants in the Lublin region. Acta Univ. Lodz. Folia bot. 13: 199-208. (in Polish)

Jackowiak B., 1990. Antropogeniczne przemiany roślin naczyniowych Poznania. / Anthropogenic changes of the flora of vascular plants of Poznań. Wyd. Nauk. UAM, Poznań, ser. Biol. 42: 1-232. (in Polish)

Jędruszczak M., Owczarczuk A., 2006. Flora chwastów w uprawach roślin okopowych w strefie ochronnej Narwiańskiego Parku Narodowego. / Weed flora in root crops in the buffer zone of the Narew National Park/. Pam. Puław. 143: 87-96. (in Polish)

Kondracki J., 2002. Geografia regionalna Polski. 441. Państwowe Wydawnictwo Naukowe, Warszawa. (in Polish)

Korczyński M., 2006. Przekształcenie flory segetalnej Tucholskiego Parku Krajobrazowego. / Segetal flora transformations in the Tuchola Lantscape Park. Pam. Puł. 143:97-104. (in Polish)

Korniak T., 1992. Flora segetalna północno-wschodniej Polski, jej przestrzenne zróżnicowanie i współczesne przemiany. / Segetal flora of north-eastern Poland, its special differentiation and current changes. Acta Acad. Agricult. Tech. Olst. Agricult. 53, Suppl. A: 5-76. (in Polish)

Korniak T., 1998. Ginące i zagrożone gatunki flory segetalnej w północno-wschodniej Polsce. / Endangered and threatened species of the segetal flora in north-eastern Poland. Acta Univ. Lodz. Folia bot. 13: 43-50. (in Polish)

Krajowa strategia ochrony i umiarkowanego użytkowania różnorodności biologicznej. Ministerstwo Środowiska. / The National Strategy of Protection and Moderate Use of Biological Diversity, Warszawa. 2003. (in Polish)

Li ro A., 2002. Programy rolnośrodowiskowe - Instrument ekologizacji gospodarki rolnej IUCN - TheWorld ConservationUnion. Fundacja IUNCPoland. WWF Światowy Fundusz na rzecz Przyrody, Warszawa, 2002, (in Polish) 
Mirek Z., Piękoś-Mirkowa H., Zając A. \& Zają c M., 2002. Flowering plants and pteridophytes of Poland - a checklist. - [In:] Mirek (ed.) Biodiversity of Poland 1: 42. W. Szafer Institute of Botany, Polish Academy of Sciences, Kraków.

Ratuszniak I., Sobisz Z ., 2006. Flora segetalna Słowińskiego Parku Narodowego. / Segetal flora of the Słowiński National Park. Pam Puław. 143: 125-134. (in Polish)

Rut kow sk i L., 2007. Klucz do oznaczania roślin naczyniowych Polski niżowej. Państwowe Wydawnictwo Naukowe, Warszawa : 814. (in Polish)

Siciński J.T., 2003. Agrofitocenozy dorzecza środkowej Warty i Bzury - stan, dynamika i zagrożenia. / Agrophytocoesoses of the Middle Warta and Bzura River systems - the present state, dynamics, and threats. Wyd. Uniw Łódz.: 1-55. (in Polish)

S okołowski A.W., 1989. Ubożenie roślinności segetalnej i problem jej ochrony. Parki Narodowe i Rezerwaty Przyrody. 1:45-50. (in Polish)

Sokołowski A. W., Kot J., 1996, Przyroda województwa suwalskiego, Suwałki.: $72-73$ (in Polish).

Sołtys A., 2006. Rzadkie i zagrożone gatunki chwastów segetalnych w Ojcowskim Parku Narodowym i jego otulinie. / Rare and threatened segetal weeds in the Ojców National Park and its protection zone. Pam. Puław. IUNG Puławy, 143: 173-181. (in Polish)

Trąba Cz., Ziemińska M.,1998. Stan gatunków chwastów segetalnych uważanych za zagrożone w otulinie Roztoczańskiego Parku Narodowego. / The state of threatened segetal species in the surrounding area of the Roztocze National Park. Acta Univ. Lodz. Folia bot. 13: 265-272. (in Polish)

Warcholińska A.U., 1994a. List of threatened segetal plant species in Poland. [In:] S. Mochnacky, A. Terpo (eds). Antropization and environment of rural settlements. Flora and vegetation. Proceedings of International Conference. Sattoraljaujhely: 206-219.

Warcholińska A.U., 1994b. Zmiany roślinności segetalnej Równiny Piotrkowskiej w ostatnich 22 latach. Cz. III. Zbiorowiska chwastów ściernisk. / Changes of segetal vegetation of the Piotrków Plain during the last 22 years. Part III. Weed communities of stubble fields. Acta. Agrobot. 47 (1): 55-65.

Rozporządzenie Ministra Środowiska, z dnia 9 lipca 2004 r. w sprawie gatunków dziko występujących roślin objętych ochroną (Dz. U. z dnia 28 lipca 2004 r.) na podstawie ustawy z dnia 16 kwietnia 2004 r. o ochronie przyrody (Dz. U. Nr 92, poz. 880). (in Polish)

Zając E. U., Zając A., 1975: List of archaeophytes occurring in Poland. Zesz. Nauk. UJ, 395, Prace. Bot. $3: 7-16$
Zają c A., 1979: The origin of the archaeophytes occurring in Poland. Habibit. Thesis 29,UJ: 5-213.

Zając M., Zając A., 1992. A tentative list of segetal and ruderal apophytes in Poland. Zesz. Nauk. UJ. Prace Bot. 24:7-23.

Zając A., Zając M., Tokarska-Guzik B., 1998. Kenophytes in the flora of Poland: list, status and origin. [In:] J. B. Faliński, W. Adamowski and B. Jackowiak (eds). Synantropization of plant cover in New Polish research. Phytocenosis 10. Suppl. Cartogr. Geobot. 9: 107-116.

Zarzycki K., Kaźmierczakowa R., 1993. Polska czerwona księga roślin. Inst. Ochr. Przyr. PAN, Kraków: 9-310. (in Polish)

Zarzycki K., Szeląg Z., 2006. Red list of the vascular plants in Poland. [In:] Z. Mirek, K. Zarzycki, W. Wojewoda, Z. Szeląg (eds). Red list of plants and fungi in Poland. W. Szafer Insitute of Botany, Polish Academy of Sciences, Kraków. : 9-20.

\section{Flora segetalna Wigierskiego Parku Narodowego}

\section{Streszczenie}

Badanie flory segetalnej Wigierskiego Parku Narodowego prowadzono w latach 2008-2010 na terenie 33 miejscowości. W uprawach zbóż, okopowych i na ścierniskach wykonano 195 zdjęć fitosocjologicznych. Ogółem w agrocenozach zarejestrowano 181 gatunków, które należały do 36 rodzin botanicznych. Najliczniejsze w gatunki rodziny to Asteraceae, Fabaceae i Brassicaceae. We florze segetalnej Wigierskiego Parku Narodowego przeważają gatunki rodzime - apofity $(53,6 \%)$ nad antropofitami. Wśród apofitów najczęściej notowano apofity łąkowe (35 gatunków) i siedlisk nadwodnych (26 gatunków). W grupie antropofitów dominują archeofity (74 gatunki), nad epekofitami (9 gatunków) i ergazjofitami (1 gatunek). Wśród analizowanych form życiowych przeważają terofity $(61,9 \%)$ nad hemikryptofitami $(27,1 \%)$ i geofitami (11\%). We florze segetalnej Wigierskiego Parku Narodowego odnotowano ponad dwukrotnie więcej gatunków krótkotrwałych $(68,5 \%)$, niż wieloletnich $(31,5 \%)$. W badanej florze wystąpiło 31 gatunków cennych przyrodniczo, o różnych kategoriach zagrożenia. Do gatunków objętych ochroną ścisłą (Dz.U. 2004 $\mathrm{Nr} 168$, poz. 1764) należą Centaurium pulchellum i Centaurium erythraea. 